\title{
Discussion of Logistics Network Optimization Problems in the Cloud Logistics Environment
}

\section{$\mathbf{R u} \mathbf{M i}^{\mathbf{1}}$}

College of Management Science and Engineering Shandong Normal University

Shandong, 250014, China

E-mail:15264173151@163.com

\section{Ming-chun Zheng ${ }^{23}$}

College of Management Science and Engineering Shandong Normal University

Shandong, 250014, China

E-mail:zhmc1630163.com

The paper mainly studies the problem of logistics optimization in the cloud logistics network environment. Firstly, it introduces the cloud logistics and sets up a platform of cloud logistics information, then introduces the function of this platform. Considering the restrain of warehousing, forwarding, distribution and transport capacity of the entire logistics system in combination with the running mechanism of cloud logistics system, it studies the vehicle routing optimization problem of logistics network in the dynamic environment that random variation of customers' needs and random service time is necessary, and builds a mathematical model in this environment, then uses an adaptive large neighborhood heuristic search algorithm to solve this problem.

\footnotetext{
${ }^{1}$ Speaker

${ }^{2}$ Corresponding Author

${ }^{3}$ This work was supported by the Natural Science Foundation of China (NO.: 61402266) and Natural Science Foundation of Shandong Province (NO.: ZR2012MF013).
} 


\section{Introduction}

"Cloud logistics" provides a train of new thought to solve the logistics problem namely in the internet of things[1], cloud computing and other information technology and platform's support in combination with the personalized needs of customers[2], conforms a dispersed and a number of logistics services provided by different logistics service providers. It is integrated by a new service concept and service platform to form a new cluster logistics services, create the new customer value and customer experience.

The logistics servic is based on the cloud logistics information platform. It can plan the logistics public information platform and the management platform, apply to the e-commerce distribution system, in which, the logistics public information platform is for customer service layer to get powerful information and the logistics management platform is for the user operation layer to quickly improve the work efficiency of logistics and relevant enterprises and extend out a wider range of business area, as shown in Fig. 1. Firstly, the cloud information service platform summarizes the whole customers' information order from the demand side, integrates the order information, reuses of the supply-side logistics management platform to integrate the scattered carrying capacity of small logistics company, small and medium-sized express company can access the customer information by visiting the cloud logistics information service platform, and can also pick up and deliver goods by it[3].

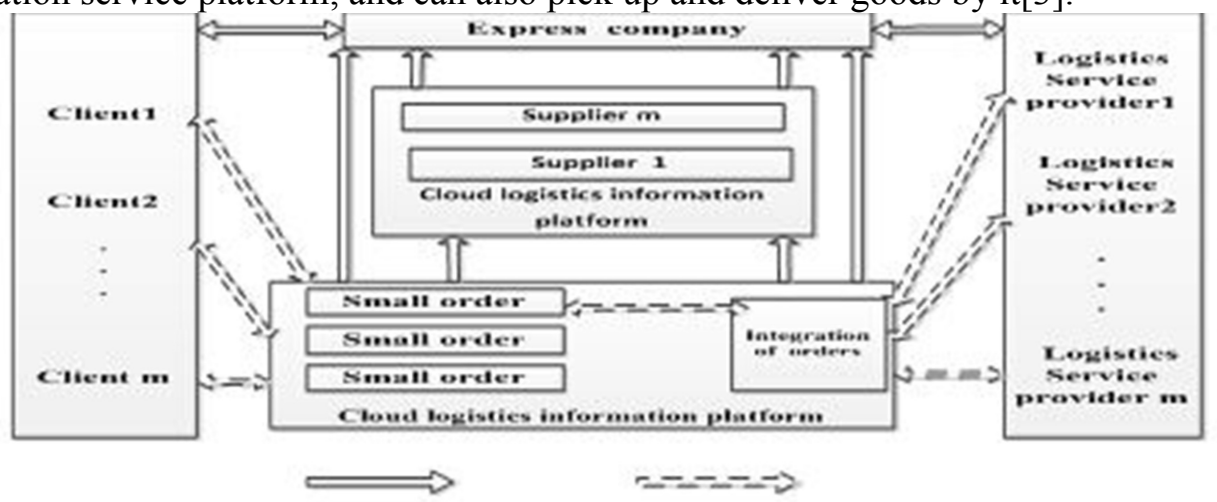

Figure 1: logistics data flow

\section{Study of Random Vehicle Routing Optimization Problem}

Under the background of logistics information platform, build the vehicle routing information service platform, as shown in Fig. 2.

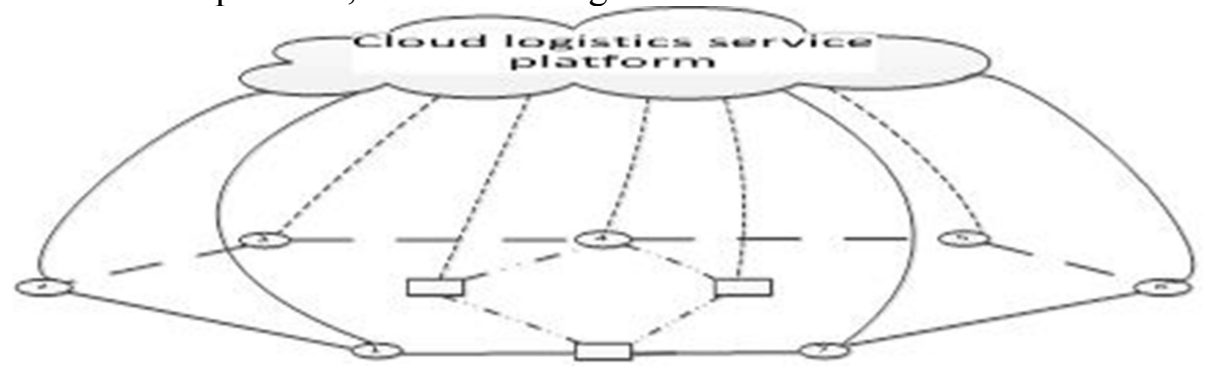

Figure 2: Client $\square$ logistics center

The cloud logistics information platform can accept all customer shipment order requests. Sometimes, the customer may temporarily increase or cancel the number of good delivery[4], which would result in the demand uncertainty.Although the company knows the number of goods for delivery with the help of cloud logistics service control center, it will change; in this sense, the cumulative total pick-up will fail to pick up service to normal operation in the planning pick-up way in advance. At this point, the platform can reasonable arrange the other 
alternative express company to collect the order according to the logistics information in the cloud logistics information network. The cloud logistics information platform can quickly make arrangement again under the environment of customer increase or cancellation of their order so as to make the whole logistics system to be well operated in an orderly way.

\section{Problem Description and Solution}

We will consider the pickup vehicle routing problem with the following characteristics in this paper:

(1)we can know that the capacity of car which performs task is limited and the loading service time for each customer;

(2)the vehicle must leave and return back to the parking lot in the prescribed time limit and each car executes only once distribution task for one day;

(3)there is no exchange of goods between customers, a route with pickup task has not prescribed the service order;

(4)a customer can only be serviced by one car;

(5)the goods that customer supply must be sent back to the distribution center;

(6)the ultimate goal of problem is to minimize the sum of all traveling vehicles and the fixed vehicles.

There is a road from $V_{i}$ to $V_{j}, X_{i j k}:\left\{\begin{array}{l}1, \\ 0,\end{array}\right.$ there is no road from $V_{i}$ to $V_{j}$. As the decision variables of problem; Q: the solution of problem, as vector of $X_{i j k} ; \phi(Q)$ : the traveling costs of all cars; $\varphi(Q)$ : due to the service failure on each path, the additional cost produced by the solution of $\mathrm{Q} ;{ }^{\zeta}(Q)$ : due to the service failure on each path, the additional return path travel expenses produced by the solution of $\mathrm{R} ;{ }^{\theta}(Q)$ : due to the service failure on each path, in order to meet the demand of customer peak. And the fees of direct service on each vertex equal to the cost back and forth between the warehouse and vertex multiplied $\beta$ the penalty factor; ${ }^{\alpha}$ : the service window time of custom $i$.

Model:

$$
\begin{gathered}
\min \{\phi(Q)+\mathrm{E}(\varphi(Q))\} \\
\left.=\min \left(\sum \sum \sum \operatorname{SijkXijk}+\mathrm{E}(\zeta(Q)+\theta(Q))\right)\right) \\
\text { s.t } \sum \sum X i j k=1 \quad(V j \in V) \\
\sum X i j k=X j i k \quad(V i \in V, k \in K) \\
\sum X i k=\sum X k i \quad k \in K \\
\sum \Sigma X i j k=X j i k=m \\
\mathrm{P}\{\Sigma \delta i \Sigma X i j k \leq 2 \mathrm{~W} \kappa\}<\mu \quad k \in K \\
0 \quad\{V i \in V, V j \in V, k \in K)
\end{gathered}
$$




$$
\begin{array}{cc}
h t_{i k}^{d} \quad \alpha_{i} & (V i \in V, k \in K) \\
X i j k \in\{0,1\} \quad & \{V i \in V, V j \in V, k \in K) \\
& Q=\left(x_{i j k}\right)
\end{array}
$$

Type (3.1) is the objective function of problem. There are two parts: the total cost of transportion along the path by logistics delivery vehicle and the expected total extra cost produced by service failure on vehicle routing under random demanding. Constraints (3.2) and (3.3) ensure the transportion and only one car service for each customer vertex. Constraint (3.4) ensures that each car to be used only once. Constraint (3.5) ensures each car in the vehicle collection is used.Constraint (3.6) describes that the each vertex has its own service time window, so the planned logistics vehicles must be completed pick-up task within the time window.Constraint (3.7) shows that the decision varies with the binary varying. Type (3.8) shows that the solution $\mathrm{Q}$ of problem is vector. Considering the random demand, the services demanding for vehicles along the service path are likely to exceed the car's capacity, which will fail the service.

Solution: the paper used an adaptive large neighborhood heuristic search algorithm to solve above problem [5]. Firstly, it used an algorithm constructed by a kind of effective heuristic initial solution to get a good initial solution. It chose a heuristic delete derived algorithm to "destroy" the current solution each iteration, and selected a heuristic insert derived algorithm to "fix" the damaged solution. The operation of "destruction" and "fix" aimed to develop further solution space effectively.Accepting the new solution and terminating algorithm search are confirmed by the defined criteria of search framework. Large neighborhood researches, refer to deletion of the q customer vertex by a heuristic derived algorithm from path, reinsert the $q$ customer peaks by a heuristic derived algorithm from path, produce a search procedure of new solution. Initial solution construction algorithm arrange all the customers' peaks from small to large according to the demand value that customer peak expected firstly, then began insert the vertex orderly from the first into the best position of all paths, ensure that constraint (3.6) is established meanwhile. In order to generate initial solution quickly, it calculates the insert charges only considering the part identification in the objective function, which means the path travel expenses. The insert costs that customer vertex lie in i position on the path can search out the first unpaired path, and some requirements on peak can be inserted as the minimum cost on this path. These requirements when the insert should ensure that the constraint (3.5) have been established. To be simple, it considers the tests that have limited the number percentage as demanded, such as 0.1 as the initial value and the growth to try a finite numerical value[6].

Although we have established a model based on integer linear programming, but when it comes to smaller problems, we can directly solve it by integer linear programming model, get the global optimal solution; therefore, we use software Lingo 8.0 to write the implement program of algorithm and use an example to do the calculation test.

\section{Application Examples}

In order to test the above algorithm, we selected a vehicle routing problem example that collected by a cargo warehouse (No.0) and 15 clients (No.1, 2,,15) in one city, as shown in Fig. 2 . We assume that the distance between the distribution center and various customers is known. Each customer all have taken goods demand, the demand is known (in Table 1), the distribution center has five cars now, the car capacity is 55, the cars are the same and have the same speed $(30 \mathrm{~km} / \mathrm{h})$ on the road, the total time per vehicle makes the loading service for a customer is 5 minutes. The cost that per car run RMB1/km, and the total time per car from its departure from the distribution center and its return back every day is no more than 4 hours. Vehicles depart from 
fixed warehouse and return to warehouse after completing task every day. Perfect highway traffic system (between customer and customer, customer and warehouse) is known (directly); the time that vehicles take has no change (not consider uncertain factors such as congestion).

\begin{tabular}{|l|l|l|l|l|l|l|l|l|l|l|l|l|l|l|l|}
\hline $\begin{array}{l}\text { Client } \\
\text { No. }\end{array}$ & 1 & 2 & 3 & 4 & 5 & 6 & 7 & 8 & 9 & 10 & 11 & 12 & 13 & 14 & 15 \\
\hline Supply & 13 & 10 & 16 & 9 & 13 & 4 & 9 & 9 & 9 & 6 & 16 & 7 & 15 & 8 & 15 \\
\hline
\end{tabular}

Table 1: Distance Matrix between Distribution Center and Customer (unit: $\mathrm{km}$ )

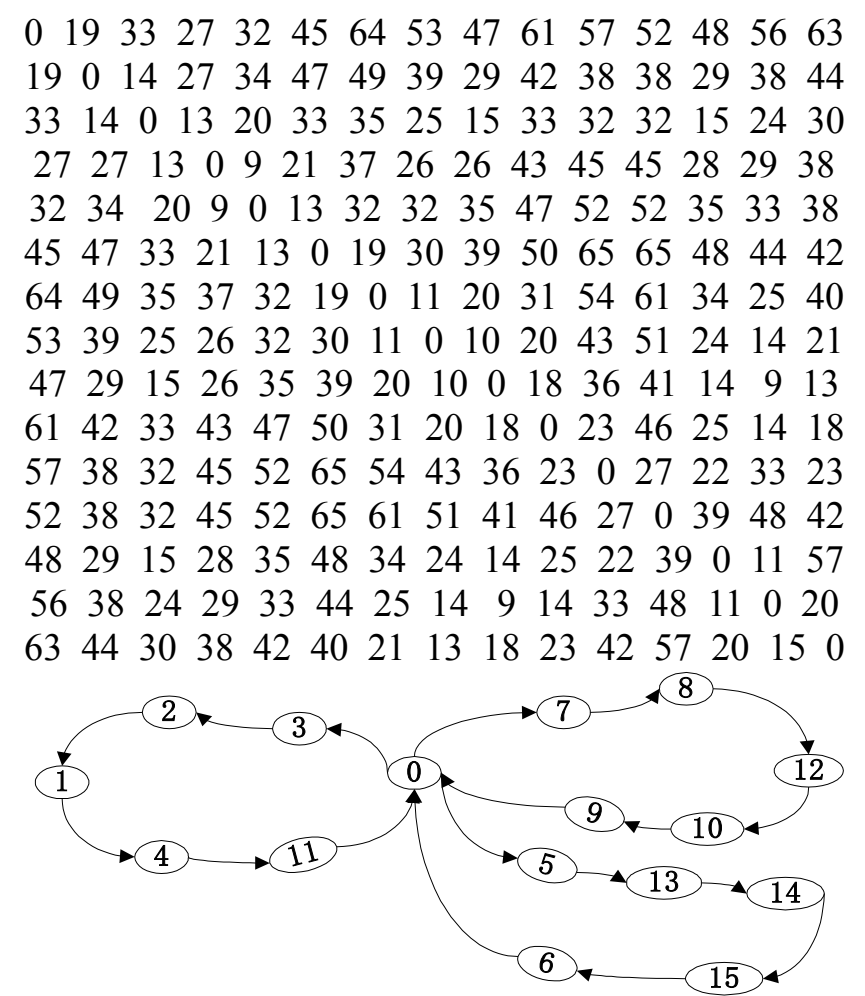

Figure 3: Path result

\begin{tabular}{|l|l|}
\hline Vehicle number & Transport path \\
\hline 1 & $0-3-2-1-4-11-0$ \\
\hline 2 & $0-6-15-14-13-5-0$ \\
\hline 3 & $0-7-8-12-10-9-0$ \\
\hline
\end{tabular}

Table 2: Calculation Results

Directly use the Lingo software to program, solve the integer linear programming model and get all the optimal solution model (as follows figure 3): the total cost is RMB285 with 3 cars demanded. Each car's running route can be seen in Table 2 and Fig. 3; at meanwhile, we are using $\mathrm{VC}++$ to write a calculation procedure based on the heuristic algorithm, and use the program to calculate path, we can also get the results in Table 2 and Fig. 3.

\section{Conclusion}

It can be concluded that the time and uncertainty demand vehicle routing optimization problem in cloud logistics environment is a typical NP-hard problem. The cloud logistics service platform can use the adaptive large neighborhood heuristic search algorithm to solve the problem directly when the logistics companies has many clients; and the solution of this 
problem can be effectively applies to real logistics system and practical problem, which will optimize the whole logistics network.

\section{References}

[1] R. A. Russell, W. C. Chiang, Scatter search for the vehicle routing problem with time windows [J]. European Journal of Operational Research, 169(32):606-622(2006).

[2] F. Alfredo, T. Montane, R. D. Galvao. A tabu search algorithm for the vehicle routing problem with simultaneous pickup and delivery service [J]. Computers and Operations Research, 2006, 33(01): 595-619.

[3] R. K. Cheung, D. Hang. Multi-attribute label matching algorithms for vehicle routing problem with time windows and back hauls [J]. IIE Transaction, 2003, 35(23): 191-205.

[4] C.-G. Lee, A. Marina, A shortest path approach to the multiple-vehicle routing problem with split pick-up[J]. Transportation Research, 2006, 40(78):265-284

[5] M. Ergun. Pickup and delivery with split load [J].Transportation Science, 2008, 42(1):32-43.

[6] G. Laporte, F. Musmanno. An adaptive large neighborhood search heuristic for the capacitated routing problem with stochastic demands [J].Transportation Science, 2010, 44(1):125-135. 